\title{
s Editorial Perspective: Pathological social withdrawal during in adolescence: a culture-specific or a global phenomenon?
}

\author{
Tim M. H. Li and Paul W. C. Wong
}

Department of Social Work and Social Administration, The University of Hong Kong, Hong Kong, China

Impairing patterns of long term adolescent social withdrawal and self-exclusion, including nonattendance at school or work, and minimal social contact, have been identified as a significant clinical and social problem in Japan since the late 1990s, where it is termed hikikomori (Kato et al., 2012). It has been estimated that there were about one million young 2 people with this condition in Japan (Saito, 1998).

Hikikomori is associated with a range of problems at an individual level; for instance, histories of trauma (Hattori, 2006), and developmental problems are

3 common in childhood (Koyama et al., 2012). Social and psychological impairment are found later on with an increased risk of mood, anxiety, psychotic, personality, and developmental disorders (Kondo et al., 2013). At the family level, one study found that about $29 \%$ of hikikomori reported having made physical assaults on their parents (Hattori, 2006). At the societal level, a prolonged withdrawal period leads youth people to become socially disengaged and unskilled individuals and this can reduce available human capital-with the impact of this felt especially in aging societies (Toivonen, Norasakkunkit, \& Uchida, 2011). The phenomenon of hikikomori has become increasingly worrying in Japan because the "first generation" of socially withdrawn youths who seclude themselves in their parents'homes is now approaching middle age, and there are concerns about the social and economic consequences for Japan as their parents retire and pass away. As a consequence, Japan may be faced with the problem of integrating a large group of disengaged and unskilled individuals into society (Mindthesciencegap.org, 2012).

Hikikomori was once thought to be a special Japanese phenomenon perhaps shaped by particular cultural practices and historical challenges (Toivonen et al., 2011). However, there are increasing numbers of reports of this, or similar, phenomena in other societies (Kato et al., 2012). For instance, a study by Kato and colleagues using case vignettes of socially withdrawn youth to study cross-cultural perceptions of hikikomori with psychiatrists in Australia, Bangladesh, India, Iran, Japan, Korea, Taiwan, Thailand, and the United States and found that 239 of 247 psychiatrists recognized youth social withdrawal, of the sort seen in hikikomori, as a social and clinical phenomenon in their local populations. However, at this stage, the prevalence and degree of impairment associated with extreme social withdrawal in nonJapanese societies has not been estimated. In this article, we argue that there is a potential threat that if this phenomenon remains unrecognized and understudied in non-Japanese cultures at this early stage in its evolution, other middle-and-high-income countries may face the same problems in the future that Japan is currently facing.

Youth disengagement, similar though not exactly analogous to hikikomori, has also been observed in some high-income Western societies. While these have been given different names and conceptualizations, they all include an element of youth disengagement. For example, 'NEET' has been used in the United Kingdom to signify young people "not in employment, education, or training". The term "adultolescent" has been used in the United States to describe young people living with their parents who do not embrace the life of growing independence typically seen in the period of emerging adulthood. A recent large-scale empirical study covering 11 European countries has found that youth who have high media use (i.e., Internet, TV, and videogames use for reasons not related to school or work), more sedentary behavior, and reduced sleep, represent "invisible" risks (Carli et al., 2014). Carli and colleagues argued that this group of youth was traditionally seen as "normal" but, they in fact exhibit worrying signs of "invisible" risk for psychopathology and suicidal behavior. While we are cautious about suggesting that the hikikomori phenomenon of youth social withdrawal observed in some Asian societies is equivalent to the phenomenon of European youth with "invisible" risk, we would like to draw attention to possible overlapping concerns emerging in various parts of the world, especially within middle-andhigh-income countries where youth unemployment is prevalent and the social ladder is becoming more difficult to climb to upper steps for the younger individuals.

Although there has been an increase in research on youth social withdrawal in recent years, our understanding of this phenomenon is hindered by a number of factors. Specifically, there is a lack of a concrete definition and clinical criteria; and studies use unrepresentative samples and nonrigorous research methodologies. To our understanding, only two systematic reviews have been conducted on this 
topic. One focused on studies conducted in Japan (Teo \& Gaw, 2010). A second, covered both studies from Japan and other societies (Li. \& Wong, 2015) including, Hong Kong, Korea, the United States, and Oman. Both reviews consistently found that: (a) only a handful of studies report reliable data on the prevalence of youth social withdrawal. Two studies have found comparable rates at about $1-2 \%$ in Japan and Hong Kong (Koyama et al., 2010; Wong et al., 2014); and (b) there is a lack of consensus on the definition of youth social withdrawal (Li. \& Wong, 2015; Teo \& Gaw, 2010). More, three core features are commonly recognized: (i) the degree of social disconnectedness; (ii) the place of withdrawal, and (iii) the duration of withdrawal.

The review by Teo and Gaw (2010) review focused on psychopathology and suggested that in the majority of cases, social withdrawal had been diagnosed as a variety of more commonly observed psychiatric disorders including schizophrenia, social anxiety disorder, major depressive disorder, and personality disorders, such as schizoid personality disorder or avoidant personality disorder. However, a notable subset of cases with substantial impairment did not meet the criteria for any existing psychiatric disorder. Given this, and in the hope of leading to better monitoring of prevalence, research into, and treatment of hikikomori Teo and Gaw proposed that, severe and/or acute social withdrawal, could be included in future DSM as a new psychiatric disorder. Key to their argument was the fact that "social withdrawal and avoidance are not better accounted for by another mental disorder, such as social phobia (e.g. avoidance of social situations because of fear of embarrassment), major depressive disorder (e.g. avoidance of social situations as a reflection of neurovegetative symptoms), schizophrenia (e.g. isolation because of negative symptoms of psychosis), or avoidant personality disorder (e.g. isolation because of fears of criticism or rejection" (Teo \& Gaw, 2010, p.447).

There is currently limited evidence of what factors might provoke social withdrawal behavior of this sort. The review by Li. and Wong (2015) summarized and categorized clinical, psychological, familial, and social factors leading to youth social withdrawal behaviors. Specifically, they identified the importance of psychological factors relating to psychological dependence of young people. Family structure and system dynamics factors were also important. Extrafamilial factors including education systems and school environments were identified. Societal values and expectations on young people were also related to youth social withdrawal behavior.

In terms of clinical interventions, a narrative review by Chan and Lo (2014) identified ways in which Japanese youth with social withdrawal behavior might be helped and supported. They described the potential value of job training, online counseling, psychotherapy and residential care as well as horse therapy. However, the evidence relating to such treatments for this group is almost nonexistent. In Li. and Wong (2015) review, only one empirical evaluation of a home visitation intervention conducted in Korea (Lee, Lee, Choi, \& Choi, 2013) could be identified.

Overall, there is currently a lack of research on this growing phenomenon that can help to explain its development and drive future research and policy and practice. Youth social withdrawal is a culturally and conceptually complex issue and investigating it is an intrinsically challenging issue because of its hidden nature.

Any simplified view explaining this phenomenon, however, is likely to be inadequate. For example, youth social withdrawal is sometimes intuitively attributed to Internet addiction. Some studies have demonstrated a correlational relationship between youth social withdrawal and number of Internet addiction symptoms (Wong et al., 2014), but there is no longitudinal study demonstrating a causal relationship between them. By narrowing the focus of the search for explanations to Internet addition among socially withdrawn youths one may overlook other equally important factors like bullying, sexual and self-injurious behavior (Wong et al., 2014).

Youth social withdrawal is likely to, in fact, involve a complex interplay between psychological, social, and behavioral factors. It is also certainly heterogeneous. On the basis of the current level of scientific evidence, we speculate that there are, in general, three types of socially withdrawn youths: (i) overdependent, (ii) maladaptive interdependent, and (iii) counterdependent.

We propose that the overdependent type grow up in overprotective families in which they fail to undergo healthy psychosocial development, such as learning how to trust people and gain autonomy. Because their families provide adequate material resources, there is little motivation to grow independently and hence a habit of overreliance on parental support is developed. The maladaptive interdependent type, we think, are the product of dysfunctional family dynamics which leads to an inability to learn basic interpersonal skills at home. This leads to unsatisfactory relationships with peers, a vulnerability to bullying and peer rejection at school. The counterdependent socially withdrawn type is hypothesized to be burdened by high parental achievement expectations - which is associated with high academic pressure and careerrelated stress. Such individuals are likely to spend much time studying and planning for the future, but their subsequent unemployment and the lack of opportunity leave them frustrated. Thus, they withdraw from others to struggle for personal growth. This classification of putative types of youth social withdrawal, although in need empirical validation, we believe, has the potential to provide a structure that can help with the development of new interventions, and clinical treatments. 
The phenomenon of youth social withdrawal seems to have become a particular concern in some high-income countries because of their aging populations and the associated structure of the productive workforce. Unlike traditional at-risk youths, who engage in substance use and addiction, risky sexual behaviors, and delinquency issues that are relatively "visible" to parents and health professionals, socially withdrawn youths and their issues seem "invisible" or at least less apparent and can be easily overlooked or trivialized by the traditional perspective of what conceive as youth risks. Moreover, some aspects of societal change in high-income countries (Bor, Dean, Najman, \& Hayatbakhsh, 2014), may lead recent cohorts of young people to develop more "invisible" risks. From a research perspective, undoubtedly, we need more observational studies employing rigorous quantitative and qualitative research methodologies to understand this emerging youth issue. Advances in understanding of this issue are likely to come through increased testing of theoretical models supporting by both qualitative and quantitative data so that causal, rather than just correlational, processes can be identified. The key methodological obstacles in the study of youth social withdraw remain that the hidden nature of such behavior and the motivation to conceal such behavior by both the youths and their parents. These problems are likely to hinder research on this issue if future research rely only on traditional research methodologies; however, recent methodological advances, for examples, web-based surveys delivered through social media platforms, and text data mining of weblogs written by potential withdrawn youths, may offer novel alternative or solutions to some methodological obstacles. Also, the perspectives of parents, family members, school teachers, friends, and professionals on social withdrawal are needed to formulate a more complete picture of the issue. From a clinical perspective, it is premature, or maybe harmful, to pathologize this new youth issue when evidence-based intervention is almost nonexistent. More efforts are needed to develop reliable assessment tools and innovative interventions to support the clinical development on this issue if we are to assist withdrawn young people to reintegrate back into our societies and reclaim their social status and momentum in life.

\section{Acknowledgements}

This work was not supported by any external funding. We thank all the colleagues from the Chinese Evangelical Zion Church, Hong Kong SAR for their efforts in providing animal-assisted interventions for withdrawn young people and allowing us to learn from their practical experiences and wisdom. The authors have declared that they have no competing or potential conflicts of interest.

\section{Correspondence}

Paul W. C. Wong, Rm 511, Jockey Teaching Tower, The Centennial Campus, The University of Hong Kong, Pokfulam Road, Hong Kong, China; Email: paulw@hku.hk

\section{References}

Bor, W., Dean, A.J., Najman, J., \& Hayatbakhsh, R. (2014). Are child and adolescent mental health problems increasing in the 21 st century? A systematic review. Australian and New Zealand Journal of Psychiatry. Retrieved from http:// dx.doi.org/10.1177/0004867414533834.

Carli, V., Hoven, C.W., Wasserman, C., Chiesa, F., Guffanti, G., Sarchiapone, M., ... \& Wasserman, D. (2014). A newly identified group of adolescents at "invisible" risk for psychopathology and suicidal behavior: Findings from the SEYLE study. World Psychiatry, 13, 78-86.

Chan, G.H.Y., \& Lo, T.W. (2014). Hidden youth services: What Hong Kong can learn from Japan. Children and Youth Services Review, 42, 118-126.

Hattori, Y. (2006). Social withdrawal in Japanese youth: A case study of thirty-five hikikomori clients. Journal of Trauma Practice, 4, 181-201.

Kato, T.A., Tateno, M., Shinfuku, N., Fujisawa, D., Teo, A.R., Sartorius, N., .. \& \& Kanba, S. (2012). Does the 'hikikomori' syndrome of social withdrawal exist outside Japan? A preliminary international investigation Social Psychiatry and Psychiatric Epidemiology, 47, 1061-1075.

Kondo, N., Sakai, M., Kuroda, Y., Kiyota, Y., Kitabata, Y., \& Kurosawa, M. (2013). General condition of hikikomori (prolonged social withdrawal) in Japan: Psychiatric diagnosis and outcome in mental health welfare centres. International Journal of Social Psychiatry, 59, 79-86.

Koyama, A., Miyake, Y., Kawakami, N., Tsuchiya, M., Tachimori, H., \& Takeshima, T. (2010). Lifetime prevalence, psychiatric comorbidity and demographic correlates of "hikikomori" in a community population in Japan. Psychiatry Research, 176, 69-74.

Lee, Y.S., Lee, J.Y., Choi, T.Y., \& Choi, J.T. (2013). Home visitation program for detecting, evaluating and treating socially withdrawn youth in Korea. Psychiatry and Clinical Neurosciences, 67, 193-202.

Li., T.M.H., \& Wong, P.W.C. (2015). Youth social withdrawal behavior (hikikomori): A systematic review of qualitative and quantitative studies. Australian and New Zealand Journal of Psychiatry. Retrieved from http://dx.doi.org/10.1177/ 0004867415581179.

Mind the Science Gap. (2012). Can culture create mental disease? The rise of "hikikomor" in the wake of economic downturn in Japan. Retrieved from http://www.mindthesciencegap.org/ 2012/11/16/can-culture-create-mental-disease-the-riseof-hikikomori-in-the-wake-of-economic-downturn-in-japan/.

Saito, T., 1998. Shakaiteki Hikikomori [Social Withdrawal]. PHP Kenkyuujo, Tokyo. (in Japanese).

Teo, A.R., \& Gaw, A.C. (2010). Hikikomori, a Japanese culturebound syndrome of social withdrawal? A proposal for DSM-5 Journal of Nervous and Mental Disease, 198, 444-449.

Toivonen, T., Norasakkunkit, V., \& Uchida, Y. (2011). Unable to conform, unwilling to rebel? Youth, culture, and motivation in globalizing Japan Frontiers in Psychology, 2, 207.

Wong, P.W.C., Li, T.M.H., Chan, M., Law, Y.W., Chau, M., Cheng, C., ... \& Yip, P.S.F. (2014). The prevalence and correlates of severe social withdrawal (hikikomori) in Hong Kong: A cross-sectional telephone-based survey study. International Journal of Social Psychiatry. Retrieved from http://dx.doi.org/10.1177/0020764014543711.

Accepted for publication: 22 May 2015 